\title{
Routine fabrication of reduced graphene oxide microarray devices via all solution processing
}

\author{
Ruben Lanche ${ }^{1,2}$, Lotta E. Delle ${ }^{1,2}$, Maryam Weil', Xuan Thang Vu', Vivek Pachauri', Walid M. Munief', \\ Patrick Wagner ${ }^{2}$, and Sven Ingebrandt ${ }^{*, 1}$ \\ ${ }^{1}$ Department of Informatics \& Microsystem Technology, University of Applied Sciences Kaiserslautern, Amerikastr. 1, \\ 66482 Zweibrücken, Germany \\ ${ }^{2}$ Institute for Materials Research, Hasselt University, Wetenschapspark 1, 3590 Diepenbeek, Belgium
}

Received 30 December 2012, revised 7 February 2013, accepted 7 February 2013

Published online 18 March 2013

Keywords dielectrophoresis, graphene, impedance spectroscopy, interdigitated electrodes

*Corresponding author: e-mail sven.ingebrandt@fh-kl.de, Phone: +49 3724 5313, Fax: +49 37245313

A method for the routine fabrication of graphene (reduced graphene oxide ( $\mathrm{r}-\mathrm{GO})$ ) based devices is reported. We describe a solution-based technique for the fabrication of the devices on alternative substrates and subsequent on-substrate processing of graphene oxide into reduced graphene oxide. As sensor platform we mainly used interdigitated gold electrodes forming microarray structures on glass substrates. Graphene oxide was site-specifically deposited onto microelectrode arrays by dielectrophoresis followed by a solution-based reduction of graphene oxide with L-ascorbic acid. In order to test the r-GO sensor stability and performance, impedance spectroscopy was used. The optimized all solution processed fabrication provides an attractive fabrication route for robust $\mathrm{r}-\mathrm{GO}$ microarray devices in a flexible, scalable and cost-effective approach.

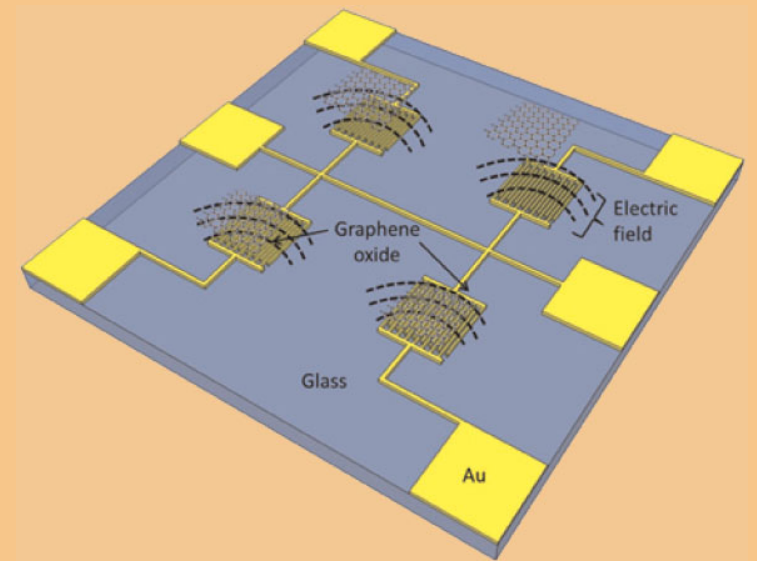

Schematics for a microarray chip based on reduced graphene oxide.
1 Introduction Recently, graphene-based devices have been extensively investigated and demonstrated as highly sensitive detectors for a variety of analytes including gases [1] and biomolecules such as glucose [2], nucleic acids $[3,4]$, proteins [5], etc. The high sensitivity of such devices is attributed to the remarkable properties of graphene as a single-atom two-dimensional lattice providing a maximized area for surface bound conduction of electron and hole charge carriers and very high charge carrier mobilities under field-effect [3, 4, 6-9]. Distinguished electrical and optical properties of graphene are being harnessed in a variety of readout approaches such as electrochemical [10], impedimetric [3], and optical [11] detection of different analytes.

The use of graphene as an active material in electrical and optical sensors has catapulted into a variety of methods to obtain thin layers of the material. The preparation methods for thin layer graphene and their manipulation on different kinds of substrates have come a long way from the erstwhile mechanical cleavage methods using a "scotch-tape" followed by a manual transfer onto a substrate [12-14]. Some of the most efficient methods for device fabrication include polymer based transfer methods of graphene single- or multi-layers grown on metal surfaces in a CVD method, 
site-specific deposition of graphene oxide from a dispersion by applying electrical fields $[12,15,16]$ or chemical trapping [17] followed by a reduction procedure for graphene oxide. Such methods are widely tested and demonstrated for fabricating a myriad of devices aimed at applications in the field of optics, electronics, sensors, reinforced and advanced materials, etc. However, cheaper and environment friendly approaches for the processing of graphene may further contribute to a "graphene based technology" for multipurpose use in daily life.

Here, solution based methods for the production of graphene oxide thin layers from the bulk offer a unique opportunity. Graphene oxide (GO), an oxide of graphene having different oxygen containing functional groups attached to "defect-sites" in the hexagonal lattice of graphene, is highly hydrophilic and therefore easier to obtain in solutions and chemical processing [18, 19]. Graphene oxide layers obtained in solution have been used for the fabrication of electrical and optical devices used as sensors for gas and liquid analytes $[1,18]$. Nanomaterials imprinting techniques such as micro-molding in capillaries (MIMIC) [20] or direct printing [21, 22] can be used as strong tools for fabrication of devices using dispersions of graphene oxide in solutions. Device fabrication methods using GO require reduction of oxidative functionalities on its surface via chemical [23], thermal [24], electrochemical [19], or photothermal [25] techniques. The reduction process induces partial restoration of the $\mathrm{sp}^{2}$ lattice where a careful control of reduction can be used to manipulate the properties of the material. The GO layers can be tuned to attain desirable electrical and optical properties for a specific use [26]. The solution based techniques for the reduction of GO are attractive as they offer an alternative to other methods, which are energy consuming and require expensive instrumentation.

In this work, we report on the fabrication of reduced graphene oxide (r-GO) based microarray electrical devices via an "all-solution-based" processing method. First, a large area microarray built of interdigitated microelectrodes (IDEs) was fabricated on silicon and on glass wafers. The GO layers obtained in the solution were transferred sitespecifically onto the microarrays by using dielectrophoresis (DEP). The technique was optimized in order to obtain an even coverage of GO layers on microarrays. Secondly, and more importantly, we used an L-ascorbic acid (L-AA) solution for the reduction of GO to r-GO on-chip in a controllable manner $[27,28]$. The reduction process of GO and hence the fabrication of r-GO devices was monitored step-by-step using electrical impedance spectroscopy (IS) [3, 29-31]. Structural, optical, and electronic characterizations suggest that the all-solution-processing method proposed here provides robust and routine fabrication of graphene (r-GO) based devices.

Using such methods avoiding high temperature steps, hazardous chemicals and gases while cutting down on the cost of instrumentation complexities may provide an important enforcement towards real application of graphene as a material and as part of different devices [27, 29, 32-34]. However, in this case, the approach provides an effective alternative for the fabrication of devices with large surface area in a robust and reproducible manner. This versatile approach may also be applied for fabricating devices on alternative substrates such as polymer materials which have particularly low melting temperatures and are not compatible to contemporary fabrication techniques $[15,16]$.

\section{Experimental}

2.1 Fabrication of r-GO microarray devices IDEs were fabricated on $\mathrm{Si} / \mathrm{SiO}_{2}$ and glass wafers by standard optical lithography and subsequent lift-off processes. Layers of Ti/Au were used for the electrodes. A Ti layer with a thickness of $15 \mathrm{~nm}$ served as the adhesion layer to promote the $\mathrm{Au}$ adhesion on the glass substrates. Interdigitated electrodes were produced on a wafer scale, where each chip consisted of $2 \times 2$ interdigitated electrode arrays. Each subunit on the chip had 44 fingers. The average thickness, length and width of the fingers were $300 \mathrm{~nm}, 1650 \mu \mathrm{m}$, and $10 \mu \mathrm{m}$, respectively. The gap between the fingers measured $10 \mu \mathrm{m}$.

Different concentrations and sonicating powers were used in order to prepare a homogenous GO solution. The best results were obtained using $25 \mathrm{mg}$ of GO flakes (Graphene Supermarket, USA) with $250 \mathrm{ml}$ of de-ionized (DI) water and sonicating for $8 \mathrm{~h}$ at maximum power $(140 \%$; Transsonic Digitals ultrasonicator, T680 DH). The solution was manually shaken every hour to redisperse the larger flakes accumulated at the bottom of the sonication vial.

All chips underwent a washing procedure with acetone, isopropanol and DI water in a $10 \mathrm{~min}$ ultrasonic bath, followed by $1 \mathrm{~min}$ in a plasma oven at $140 \mathrm{~W}$ and $0.6 \mathrm{mbar}$ oxygen to clean off remaining organic material and leave the surface hydrophilic for the even spread of the GO solution during DEP deposition. The starting parameters for DEP were based on a previously published work [15]. A final process was established where a measurement cell was used to clamp and electrically connect to the chips. A $3 \mu 1$ drop was put on each of the four IDEs subunits, a signal (5 Vpp, $3 \mathrm{MHz}$ ) was applied for $15 \mathrm{~min}$ to each IDE subunit, and the chips were allowed to dry for $1 \mathrm{~h}$ afterwards. Subsequently, the chips were given a strong wash in DI water. An HM 8130 function generator from Hameg (Livingston, Germany) was used in all DEP procedures.

2.2 Reduction of graphene oxide The GO flakes deposited on the microelectrode arrays were reduced using an aqueous L-AA (Sigma Aldrich, Germany) solution. The samples previously deposited with GO were left in $4 \mathrm{ml}$ of $10 \mathrm{mg} \mathrm{ml}^{-1}$ L-AA solution for up to 14 days and their reduction procedure was monitored concomitantly as a function of changing impedance. For comparison, the GO reduction procedure was also carried out in solution. Fifty milligrams of L-AA were used on $5 \mathrm{ml}$ of $0.1 \mathrm{mg} \mathrm{m}^{-1} \mathrm{GO}$ solution. The r-GO samples were collected over a period of 6 days and characterized using a Lambda $40 \mathrm{UV}-\mathrm{Vis}$ 
spectrometer. The devices with on-chip reduction of GO were compared with other devices that were prepared using previously reduced GO in solution.

2.3 Characterization The deposition and reduction process of GO flakes deposited on the IDEs were characterized by optical microscopy (Olympus BX51, Olympus, UK), scanning electron microscopy (Zeiss Supra 40 SEM, Carl Zeiss AG) and by atomic force microscopy (NanoScope Dimension 3100 AFM, Veeco probes, USA). The optical properties of GO were characterized by UV-Vis measurements (Lambda $40 \mathrm{UV}-\mathrm{Vis}$ spectrometer, Perkin Elmer, Germany). IS measurements were carried out using an Ivium Compactstat (Ivium Technologies, Netherlands).

\section{Results and discussion}

3.1 Site specific GO deposition Electrokinetics comprehends the study of polarizable particle movement during interaction with a non-uniform ac electric field. DEP, an electrokinetic technique, specifically corresponds to the movement of these particles towards or away from strong electric field regions. By applying different voltages and frequencies along IDEs it is possible to move and position particles [11].

It has been demonstrated [35] that the dielectrophoretic force acting on a spherical particle is given by

$$
F_{\mathrm{DEP}}=2 \pi \varepsilon_{\mathrm{m}} r^{3} \operatorname{Re}[K(\omega)] \nabla E_{\mathrm{rms}}^{2},
$$

$r$ being the particle radius, $\varepsilon_{\mathrm{m}}$ the permittivity of the suspending medium, $E_{\mathrm{rms}}$ the rms value of the electric field and $\operatorname{Re}[K(\omega)]$ the real part of the Clausius-Mossotti factor, which in the case of a homogeneous spherical dielectric particle is equal to

$$
K(\omega)=\frac{\varepsilon_{\mathrm{p}}^{*}-\varepsilon_{\mathrm{m}}^{*}}{\varepsilon_{\mathrm{p}}^{*}+2 \varepsilon_{\mathrm{m}}^{*}}
$$

where $\varepsilon_{\mathrm{m}}^{*}$ and $\varepsilon_{\mathrm{p}}^{*}$ are the complex permittivities of the medium and the particle. Further, these are related to the conductivity $\sigma$ and the angular frequency $\omega$ of the applied electric field through a standard formula:

$$
\varepsilon^{*}=\varepsilon-i\left(\frac{\sigma}{\omega}\right)
$$

Initial tests with DEP deposition revealed a voltagefrequency relation effect. If both values were chosen too high the gold in the IDEs peeled off. If the chosen values were too low the GO simply did not attach to the electrodes. In order to achieve a uniform coverage of our microelectrode arrays, the deposition method was further optimized by examining the coverage of $\mathrm{GO}$ on $\mathrm{Si} / \mathrm{SiO}_{2}$ chips under an optical microscope.

Figure 1 represents the images taken from the IDE subunits after the DEP deposition of GO flakes on $\mathrm{Si} / \mathrm{SiO}_{2}$ chips. Figure 1A shows an optical microscopy image of a typical IDE sub-unit from the chip. A fine coverage of the GO can be seen confined specifically within the area of the IDEs.
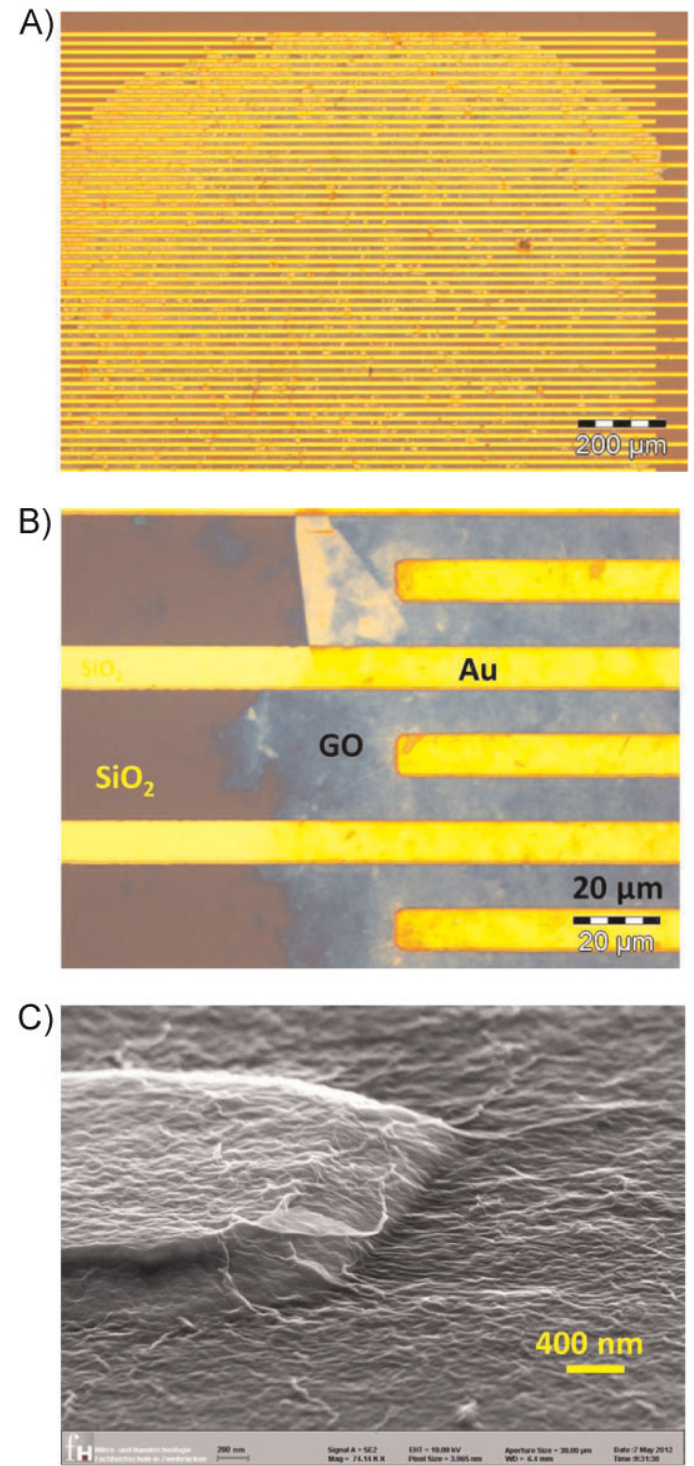

Figure 1 (online color at: www.pss-a.com) r-GO microarray devices: (A) An overview of the microarrays showing IDEs functionalized with $\mathrm{GO}$, (B) a detailed view of microelectrodes with site-specific covering of GO, and (C) a SEM micrograph of a microelectrode deposited with GO seen on an angle.

Figure 1B shows a zoomed in optical microscopy image of several IDEs at one end. The deposition of GO can easily be seen on the $\mathrm{Si} / \mathrm{SiO}_{2}$ substrate due to the change in optical reflectivity from the deposited and non-deposited surface of the chip [36]. It is clear that the procedure followed here yields a well-defined covering that ended abruptly on the borders of the IDEs. A detailed examination of GO deposition on the IDEs was done using SEM as shown in Fig. 1C, where the scanning was performed with a tilting angle of the sample. The image provides more information about the structure of the IDEs deposited with GO. The GO layer distribution and its surface is clearly visible in-between and over the IDEs. All images demonstrate an even and 

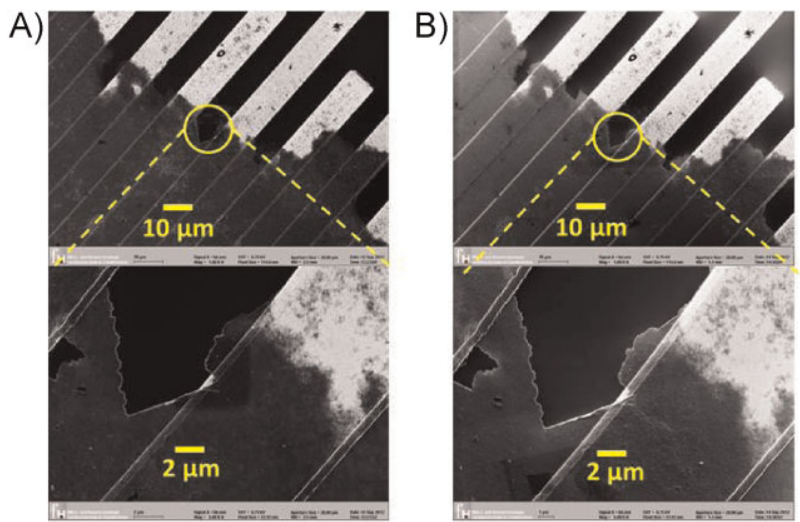

Figure 2 (online color at: www.pss-a.com) Robust r-GO microarray devices. (A) SEM image from an area of a microarray device before sonication, (B) SEM image of the same device after sonication showing no structural changes.

continuous coverage of the GO on the IDEs. Generally, a smooth coverage of the GO is advantageous as it avoids eventual changes in the electrical characteristics of the devices originating from washed off flakes during the several measurements steps performed in liquids.

The robust fabrication of microelectrode arrays functionalized with GO was tested by agitation in an ultrasonic bath for $15 \mathrm{~min}$ and comparing the morphologies of the GO layer with SEM before and after sonication. Figure 2 shows the SEM images taken on IDEs from a typical chip. It is apparent that the GO coverage on the chips was very robust as there were no visible structural changes or any peeled-off regions showing GO detachment from the IDEs.

DEP deposition of GO with the same parameters was carried out subsequently on the glass chips. Figure 3 shows a typical glass chip deposited with GO flakes. Figure 3A shows a large area AFM scan from one part of the IDE sub-unit. An even deposition of GO can be seen in the image. Figure 3B illustrates the height profiles taken from different positions of the device as depicted by the lines drawn in Fig. 3A. It is evident from the height profiles that the dielectrophoretic deposition yielded a very homogenous coverage of the GO layer across the microelectrode arrays. The thickness of the deposited layers can be deduced from the height profiles with average thickness values of about $30 \mathrm{~nm}$.

3.2 Reduction of GO Chemically GO has an abundance of oxygenated reactive groups such as epoxide and hydroxyls. The removal of hydroxyl and epoxide moieties by L-AA is performed in a SN2 nucleophilic reaction where oxygenated L-AA anion functions as a nucleophile. The attachment of these nucleophiles with the oxygenated moieties on the GO surface is followed by the release of reduction products such as $\mathrm{H}_{2} \mathrm{O}$ and therefore the repairing of the $\mathrm{C}=\mathrm{C}$ bonds in the GO lattice [28].

3.2.1 Optical monitoring The reduction procedure was monitored by using UV-Vis spectroscopy and the

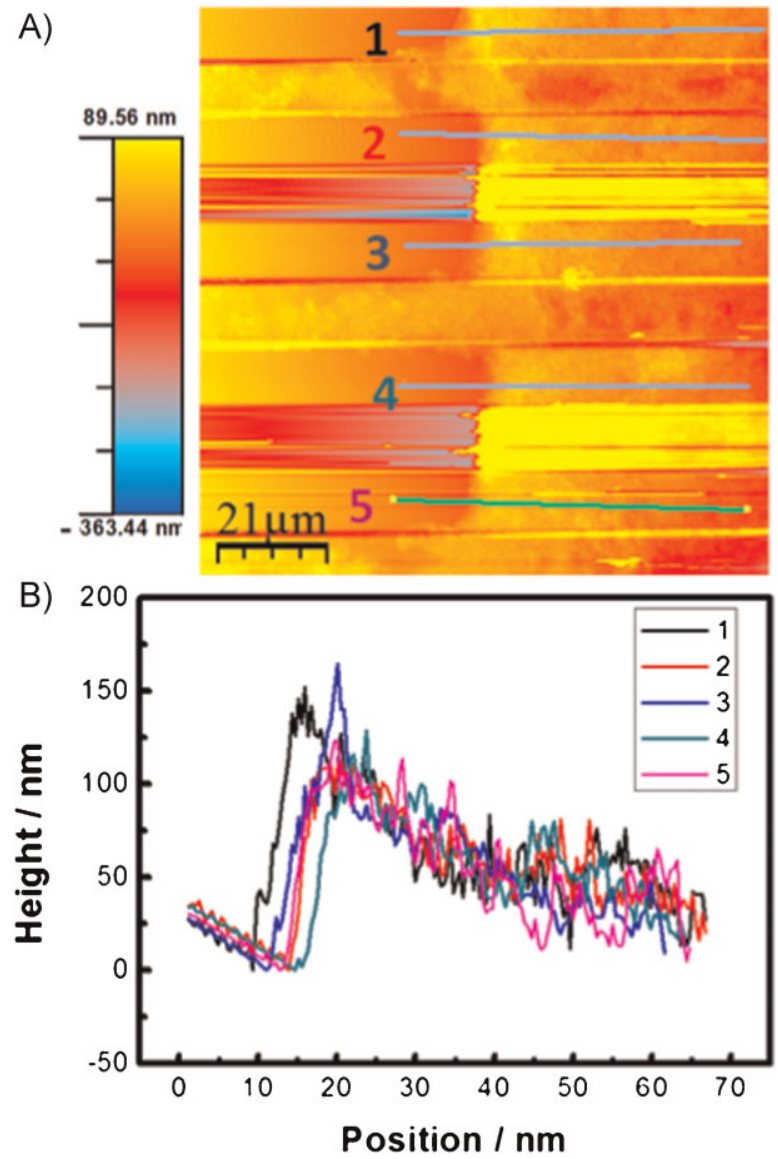

Figure 3 (online color at: www.pss-a.com) AFM characterization of r-GO microarrays. (A) AFM scan from one part of microarray fabricated on a glass wafer. Scan size: $100 \times 100 \mu \mathrm{m}^{2}$ and (B) height profiles of the GO coverage across the microelectrodes.

spectra were recorded at intermediate stages of the reduction procedure. The on-chip reduction procedure was mimicked and a concomitant optical characterization was done in order to qualitatively analyze and compare with the findings of IS. Figure 4A represents the relevant optical spectra of the GO solution recorded during the reduction procedure. Figure 4B shows optical images of the GO solution before and after 24 and $72 \mathrm{~h}$ of ascorbic acid-based reduction. It is apparent from the images that the GO solution turns blackish in color while the characteristic brown-red color diminishes gradually. The gradual reduction of oxygenated functionalities by ascorbic acid turns the GO's surface more hydrophobic resulting in coagulation and agglomeration. As shown in the picture, the GO solution completely turned black after $24 \mathrm{~h}$ and the $\mathrm{GO}$ agglomeration is clearly visible after $72 \mathrm{~h}$ as a precipitate in the solution. Figure 4A shows the optical spectra taken for the GO solution during different phases of reduction. At a wavelength of $280 \mathrm{~nm}$ the absorption peak was reduced gradually as comparable to literature $[27,33]$.

3.2.2 Impedance spectroscopic monitoring The r-GO based microarray devices were characterized with 

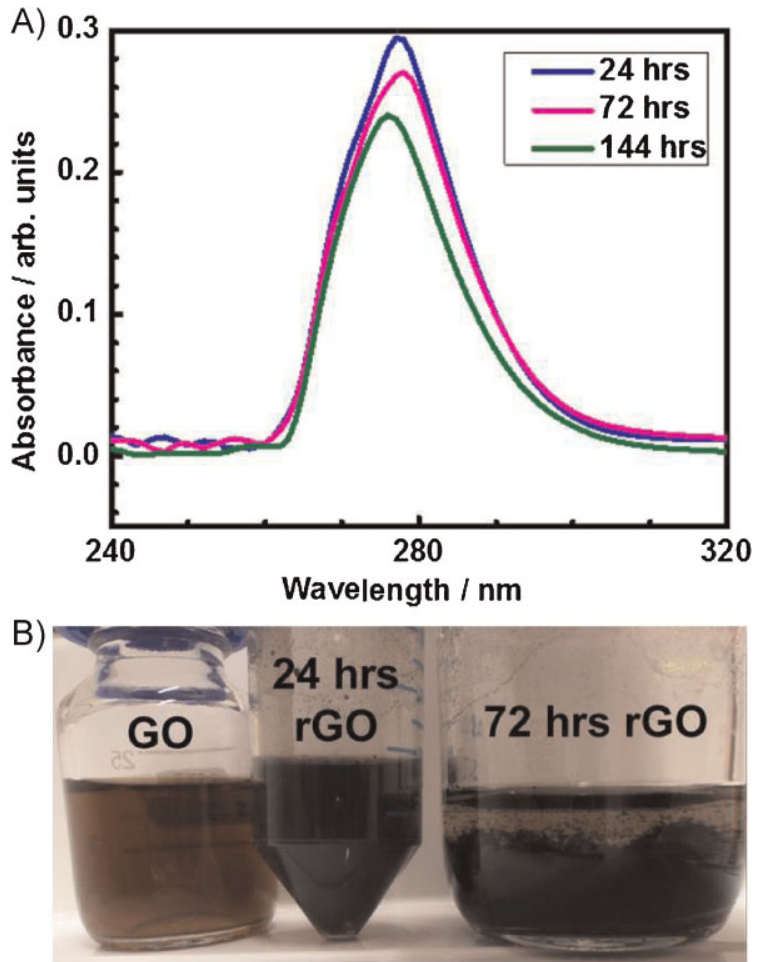

Figure 4 (online color at: www.pss-a.com) Optical characterization of the GO reduction process in solution at different stages. (A) UV-Vis spectra of r-GO solution at the different stages of reduction process. (B) Appearance of GO solution against 24 and $72 \mathrm{~h}$ after reduction $(\mathrm{r}-\mathrm{GO})$.

electrochemical IS. Figure 5 illustrates the response of typical microarray devices during successive steps of GO reduction. Figure 5B-D shows the Bode and Nyquist diagrams for typical microarray devices. As seen from the Figure 5B from the impedance versus frequency values plotted for a device, the impedance of the devices diminish as the reduction process advanced in the following days.

The frequency response of the devices plotted in the Nyquist diagram clearly shows the change from imaginary to real which corresponds to the behavior change from capacitive towards resistive after the reduction. Forty-one frequency steps between $1 \mathrm{~Hz}$ and $10 \mathrm{kHz}$ were taken in order to record these spectra while impedance values are taken at the lowest frequency measurement $(1.5 \mathrm{~Hz})$. A total of 20 chips were subjected to IS measurements here showing a similar response.

The reduction procedure was fitted using a simple resistor-capacitor parallel circuit (Fig. 5A) representing the GO layer. The respective changes in the fitted values are shown in Table 1. Although the individual readings of the impedance values were different from sensor to sensor, they were nonetheless similar enough to make a correct statistical event. Results of all experiments are summarized in Fig. 6.

Figure 6 shows a statistics where average values for the impedance of all the devices measured are plotted against the
A)
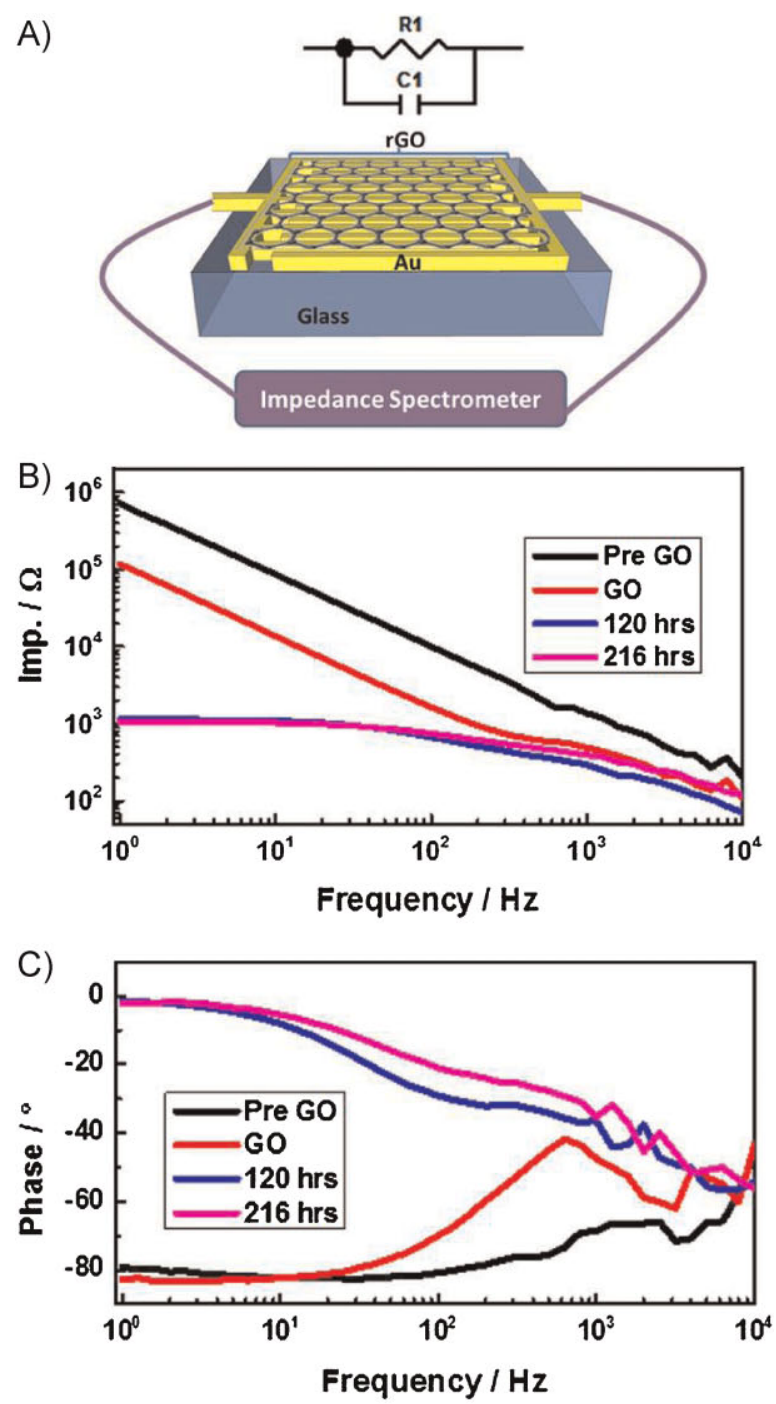

D)

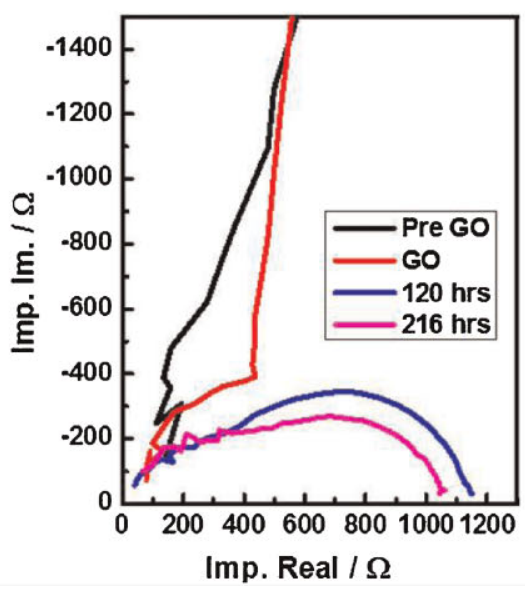

Figure 5 (online color at: www.pss-a.com) Impedance spectroscopy characterization of rGO microarray devices. Schematics for the measurement set-up and the model for the fitting of impedance and capacitance values (A), decrease in impedance (B), phase change (C) of the devices during the successive stages of GO reduction, and frequency response of the devices shown as a Nyquist plot (D). 
Table 1 Fitted circuit values for the reduction process of the DEP deposited GO flakes.

\begin{tabular}{lllll}
\hline & pre-GO & GO & 5 days & 9 days \\
\hline res. $(\Omega)$ & $1.4 \mathrm{e} 10^{10}$ & $1.4 \mathrm{e} 10^{10}$ & 1200 & 800 \\
cap. $(\mathrm{F})$ & $1.510^{-7}$ & $1.210^{-6}$ & $3.310^{-7}$ & $1.810^{-7}$ \\
\hline
\end{tabular}
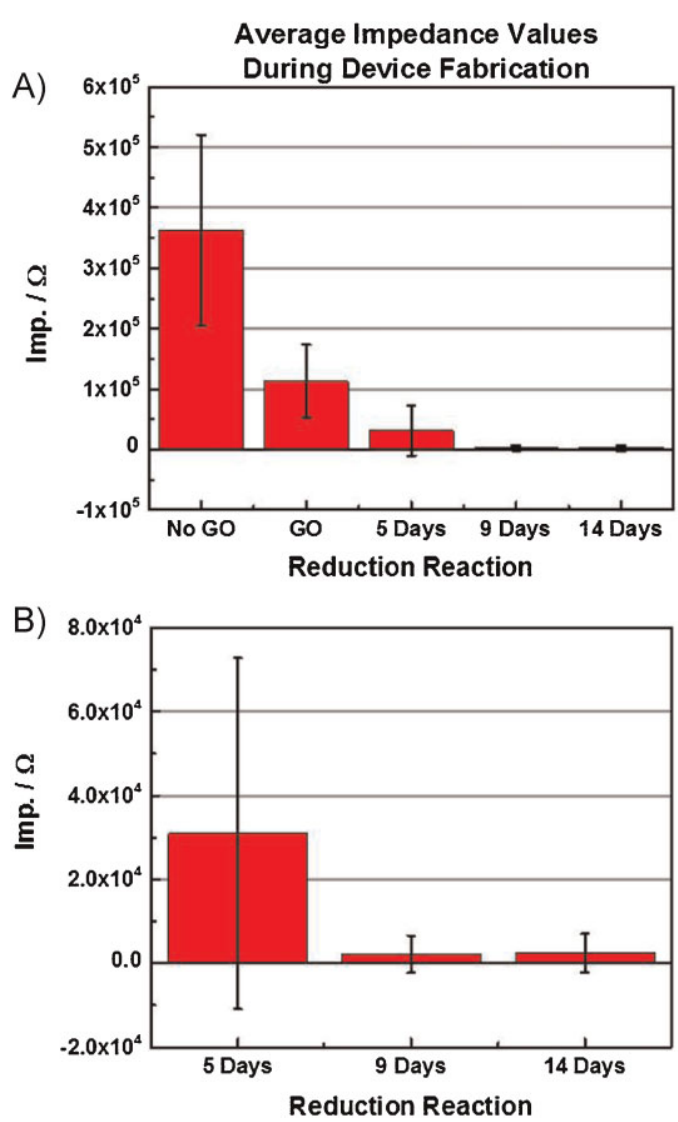

Figure 6 (online color at: www.pss-a.com) (A) Average impedance values at lowest frequency measurement $(1.5 \mathrm{~Hz})$ during device fabrication and error bars representing the standard deviation ( $n=20$ samples). (B) Close-up view from the reduction process.

reduction process. The continuous decay in the average impedance values reflects the gradual reduction of GO on-chip.

The impedance values however, after completion of the reduction process did not change overtime, suggesting for the stable and robust characteristics of chemically reduced GO on-chip. Further, as shown in Table 1, the fitting confirmed the expected reduction in capacitance due to a decrease in the dielectric constant of the GO [37-40], owing to the fact that the chip characteristics remained constant except for the change of the GO layer. It may also be discerned from Fig. 6 that there is a limit to the reduction following which there will be no change in the device. Error bars where plotted using the standard deviations.
4 Conclusions We propose a versatile all-solutionprocess method for the fabrication of graphene-based devices. As a proof-of-principle, large area microarray devices were fabricated by exfoliating graphene oxide in solution and subsequent transfer onto the microarrays using dielectrophoresis. The methods were optimized to have a homogeneous coverage of GO on the microarray devices. A benign solution-based approach was used for on-chip reduction of GO devices, which was followed by IS characterization and other techniques. The process overall facilitated results that would be beneficial for scalable fabrication of devices for different applications and offer realization of graphene-based devices on new substrates. The all solution fabrication lifts the limitation with the existing technologies and vouches for cost-effective and environmentally benign methods for efficient devices. Further experiments will focus on the expansion of this approach for realizing devices on new polymer substrates and use of devices for electrical biomolecular recognition techniques.

Acknowledgements The authors would like to thank the technical support of Erik Engelmann and Christian Neu from the mechanical workshop for the measurement cells and shadow masks. In addition Detlev Cassel for clean room support and Rainer Lilischkis (all at University of Applied Sciences Kaiserslautern, Germany) for SEM imaging are acknowledged.

\section{References}

[1] IEEE Sensors Conference, November 1-4, 2010, Waikoloa, Big Island, Hawaii (IEEE, Piscataway, NJ, 2010).

[2] H. Wu, J. Wang, X. Kang, C. Wang, D. Wang, J. Liu, I. A. Aksay, and Y. Lin, Talanta 80, 403 (2009).

[3] A. Bonanni and M. Pumera, ACS Nano 5, 2356 (2011).

[4] Z. Yin, Q. He, X. Huang, J. Zhang, S. Wu, P. Chen, G. Lu, Q. Zhang, Q. Yan, and H. Zhang, Nanoscale 4, 293 (2011).

[5] S. Mao, K. Yu, G. Lu, and J. Chen, Nano Res. 4, 921 (2011).

[6] H. Morgan, A. G. Izquierdo, D. Bakewell, N. G. Green, and A. Ramos, J. Phys. D: Appl. Phys. 34, 1553 (2001).

[7] Y. Shao, J. Wang, H. Wu, J. Liu, I. A. Aksay, and Y. Lin, Electroanalysis 22, 1027-1036 (2010).

[8] A. Bonanni, A. Ambrosi, and M. Pumera, Chemistry 18, 1668-1673 (2012).

[9] A. Barth and W. Marx, Graphene - A rising star in view of scientometrics 2008, http://arxiv.org/ftp/arxiv/papers/0808/ 0808.3320.pdf

[10] F. J. Hernandez and V. C. Ozalp, Biosensors 2, 1 (2012).

[11] R. Cheng, Y. Liu, S. Ou, Y. Pan, S. Zhang, H. Chen, L. Dai, and J. Qu, Anal. Chem. 84, 5641 (2012).

[12] Y. Liu, X. Dong, and P. Chen, Chem. Soc. Rev. 41, 2283 (2012).

[13] R. van Noorden, Nature 483, S32 (2012).

[14] K. S. Novoselov, Science 306, 666 (2004).

[15] B. R. Burg, Directing Carbon Nanotubes and Graphene: Funtamentals and Parallel Device Integration using Dielectrophoresis (ETH Zurich, Zurich, 2010).

[16] C. Chung, S. Smith, A. Menachery, P. Bagnaninchi, A. J. Walton, and R. Pethig, in: 2011 IEEE ICMTS Int. Conference on Microelectronic Test Structures (IEEE, 2011), p. 74 
[17] T. Kurkina, S. Sundaram, R. S. Sundaram, F. Re, M. Masserini, K. Kern, and K. Balasubramanian, ACS Nano 6, 5514 (2012).

[18] M. Pumera, Mater. Today 14, 308 (2011).

[19] R. S. Sundaram, C. Gómez-Navarro, K. Balasubramanian, M. Burghard, and K. Kern, Adv. Mater. 20, 3050 (2008).

[20] Q. He, H. G. Sudibya, Z. Yin, S. Wu, H. Li, F. Boey, W. Huang, P. Chen, and H. Zhang, ACS Nano 4, 3201 (2010).

[21] Y. Shao, J. Wang, M. Engelhard, C. Wang, and Y. Lin, J. Mater. Chem. 20, 743 (2010).

[22] L. T. Le, M. H. Ervin, H. Qiu, B. E. Fuchs, and W. Y. Lee, Electrochem. Commun. 13, 355 (2011).

[23] S. Stankovich, D. A. Dikin, R. D. Piner, K. A. Kohlhaas, A. Kleinhammes, Y. Jia, Y. Wu, S. T. Nguyen, and R. S. Ruoff, Carbon 45, 1558 (2007).

[24] M. Acik, G. Lee, C. Mattevi, A. Pirkle, R. M. Wallace, M. Chhowalla, K. Cho, and Y. Chabal, J. Phys. Chem. C 115, 19761 (2011).

[25] S. R. Kim, Md. K. Parvez, and M. Chhowalla, Chem. Phys. Lett. 483, 124 (2009).

[26] C. Soldano, A. Mahmood, and E. Dujardin, Carbon 48, 2127 (2010).

[27] J. Zhang, H. Yang, G. Shen, P. Cheng, J. Zhang, and S. Guo, Chem. Commun. 46, 1112 (2010).

[28] J. Gao, F. Liu, Y. Liu, N. Ma, Z. Wang, and X. Zhang, Chem. Mater. 22, 2213 (2010).
[29] A. Bonanni, I. Fernández-Cuesta, X. Borrisé, F. PérezMurano, S. Alegret, and M. del Valle, Microchim. Acta 170, 275 (2010).

[30] B. Pejcic and R. de Marco, Electrochim. Acta 51, 6217 (2006).

[31] O. Pänke, T. Balkenhohl, J. Kafka, D. Schäfer, and F. Lisdat, in: Biosensing for the 21st Century, Vol. 109, edited by R. Renneberg and F. Lisdat (Springer, Berlin/Heidelberg, 2008), p. 195.

[32] P. Van Gerwen, W. Laureyn, W. Laureys, G. Huyberechts, M. Op De Beeck, K. Baert, J. Suls, W. Sansen, P. Jacobs, L. Hermans, and R. Mertens, Sens. Actuators B 49, 73 (1998).

[33] Z.-J. Fan, W. Kai, J. Yan, T. Wei, L.-J. Zhi, J. Feng, Y.-M. Ren, L.-P. Song, and F. Wei, ACS Nano 5, 191 (2011).

[34] D. Berdat, A. C. Martin Rodríguez, F. Herrera, and M. A. M. Gijs, Lab Chip 8, 302 (2008).

[35] A. Kuzyk, Electrophoresis 32, 2307-2313 (2011).

[36] I. Jung, J.-S. Rhyee, J. Y. Son, R. S. Ruoff, and K.-Y. Rhee, Nanotechnology 23, 25708 (2012).

[37] T. T. Grove, M. F. Masters, and R. E. Miers, Am. J. Phys. 73, $52(2005)$

[38] T. Wehling, E. aşoğlu, C. Friedrich, A. Lichtenstein, M. Katsnelson, and S. Blügel, Phys. Rev. Lett. 106, 23 (2011).

[39] D. Joung, A. Chunder, L. Zhai, and S. I. Khondaker, Appl. Phys. Lett. 97, 93105 (2010).

[40] W. Fu, L. Liu, W. Wang, M. Wu, Z. Xu, X. Bai, and E. Wang, Sci. Chin. Phys. Mech. Astron. 53, 828 (2010). 